\title{
Epigenetics and its role in disease
}

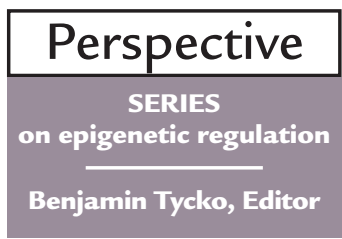

\author{
Benjamin Tycko $^{1}$ and John Ashkenas ${ }^{2}$ \\ ${ }^{1}$ Institute for Cancer Genetics, and Department of Pathology, Columbia University, New York, New York 10032, USA \\ ${ }^{2}$ Science Editor, Journal of Clinical Investigation; and Division of Medical Genetics, University of Washington, \\ Seattle, Washington 98195, USA \\ Address correspondence to: Benjamin Tycko, Institute of Cancer Genetics, Room 412, Russ Berrie Research Pavilion, \\ 1150 St. Nicholas Avenue, New York, New York 10032, USA. Phone: (212) 304-7165; Fax: (212) 304-7336; E-mail: bt12@columbia.edu.
}

The adjective "epigenetic" has been used to describe many types of biological processes, but with the evolution of epigenetics into a subdiscipline of molecular biology, its meaning has become quite focused. Although the term is sometimes used more broadly, epigenetic effects are usually taken to encompass changes in the genetic material - the genomic DNA and chromatin - that alter gene expression in a manner that is heritable during somatic cell divisions (and sometimes even in germline transmission), but that is nonmutational and therefore fundamentally reversible. The articles collected here consider in some detail the consequences of such effects for human disease phenotypes.

A major theme of the series is that lesions in human disease genes can be partly or entirely epigenetic. Researchers in this area are engrossed with the fascinating complexities of these lesions, and hints of mechanisms for both normal and pathological epigenetic regulation are emerging. There is also an obvious practical motivation to study epigenetic gene regulation in disease states: as a nonmutational and reversible process, it is, at least in principle, amenable to therapy. Agents that interfere with DNA methylation or that alter histone acetylation can erase epigenetic marks. Although the drugs studied to date may be too broad in their effects to be useful clinically, compounds that act similarly but with sequence-specific effects would be attractive therapeutic agents for certain cancers and other diseases. Furthermore, as T. Bestor (this series) argues, epigenetic effects may also account for the unexpected difficulty of developing gene therapies to treat diseases that are not, themselves, epigenetic in origin.

Among the most dramatic examples of epigenetic phenomena is the nearly complete inactivation of transcription of many hundreds of genes on the inactive $\mathrm{X}$ chromosome in female cells. In most cases, this event occurs randomly on one or the other of the $\mathrm{X}$ chromosomes in cells in normal female embryos and is maintained clonally during development, with profound influences on the expression of X-linked genetic diseases. Genomic imprinting (as discussed by E. Maher and W. Reik, this issue) and methylation-related silencing of autosomal genes (B. Tycko, this series) both show a number of instructive parallels with $\mathrm{X}$ inactivation.

Genomic imprinting is an epigenetic process that causes monoallelic gene expression by silencing or activating one of the alleles of an autosomal gene, depend- ing on the parent of origin. The resulting allelic asymmetry distinguishes imprinting from other forms of epigenetic regulation. For a number of imprinted genes, the imprint appears to silence gene expression, and in working parlance the "imprinted allele" is often identified with the silent one. Strictly speaking (and for purposes of this series), the imprinted allele should be understood to be the allele that has undergone an active epigenetic modification during gametogenesis, whether that modification ultimately causes transcription of a given gene to be activated or suppressed. However, the precise molecular changes that constitute the primary imprint are not yet known. DNA methylation continues to be a strong candidate, but whether this class of modification represents a primary or secondary cause of epigenetic silencing remains a matter of some debate. Nonetheless, methylation of CpG-rich regulatory sequences correlates closely with the imprinting of some genes, and such genes lose their allelic asymmetry in DNA methyltransferase-deficient mouse embryos.

The article by E. Maher and W. Reik (this issue) describes the imprinted genes that underlie the Beckwith-Wiedemann syndrome (BWS), a clinical condition associated with somatic overgrowth and a variable predisposition to cancer. Like $\mathrm{X}$ inactivation, imprinting can affect multiple contiguous genes within an extended chromosomal region, although the scale of the effects are very different: imprinted chromosomal domains like the one implicated in BWS are measured in megabases whereas, $\mathrm{X}$ inactivation affects a majority of the genes across much of an entire chromosome. This "domain effect" has interesting implications for the mechanism and evolution of imprinting, but it also creates a conundrum for sorting out the identities of the genes that actually contribute to the disease phenotype. For BWS, Maher and Reik show that the syndrome can be caused, independently, by lesions in at least 2 different imprinted genes, CDKN1C and IGF2, which happen to lie in the same imprinted domain. Evidence reviewed by these authors suggests that many cases of BWS are entirely epigenetic in origin; reports of monozygotic twins who are discordant for this syndrome are consistent with this view.

In contrast to BWS, Prader-Willi syndrome (PWS), a developmental and behavioral disease linked to another imprinted domain, may indeed result from altered 
expression of multiple contiguous imprinted genes. A closely linked disorder, the Angelman syndrome (AS), shows an opposite parent-of-origin dependence and can be caused by lesions in a single imprinted gene, $U B E 3 A$. Some cases of PWS and AS result from monoallelic subchromosomal deletions (paternal for PWS and maternal for AS), but, for both syndromes, there is also evidence for a class of patients whose causative chromosomal lesions are entirely epigenetic. The article by R. Nicholls will expand on these points and review the evidence for discrete DNA sequences that function as primary "imprinting control centers." His discussion will also highlight information from studies in mice or humans that point to a more general role for imprinted genes in modulating brain development and behavior.

The transcriptional silencing of the inactive $\mathrm{X}$ chromosome is propagated by both DNA methylation and histone deacetylation. Recent evidence points to a direct biochemical link between these two types of modifications, and both of them - but particularly DNA methylation - are thought to help establish and propagate imprinted states. As discussed by B. Tycko (this series), these mechanisms are also implicated in the epigenetic silencing of tumor suppressor (TS) genes in a wide array of tumor types. Epigenetic silencing of certain TS genes occurs at very early stages of multistage tumorigenesis, but this phenomenon can also affect genes that function as suppressors of invasion or metastasis. The article by Tycko discusses several hypotheses to account for gene-specific de novo methylation in cancer precursor cells. Finally, T. Bestor briefly reviews the evidence that epigenetic gene silencing evolved as a host-defense system. Bestor closes his article, and the series, with a timely discussion of transgene silencing as a cause of gene therapy failures.

In gathering together the four separate articles in this series in two successive issues of the JCI, we hope that the shared themes will create a whole that is greater than the sum of the parts. If the series stimulates new experiments, we will judge it a success. 